\title{
Carrier-to-Noise Ratio Improvement with Pulsed-Laser Readout on Magnetically Induced Superresolution by Center Aperture Detection Disks
}

\author{
Bing-Mau Chen, Bor-Wen Yang, Wein-Kuen Hwang, Tsung-Eong Shieh, and Han-Ping D. Shieh
}

\begin{abstract}
Magnetically induced superresolution by center aperture detection (MSR-CAD) using pulsed and conventional dc schemes were applied to magneto-optical disks to quantitatively evaluate readout performances. By simulation and experiments, it was found that carrier-to-noise ratio (CNR) in the pulsed-laser readout was about $2.5 \mathrm{~dB}$ higher than that in the dc readout scheme at mark size of $0.45 \cdot \mu \mathrm{m}$ or shorter. Therefore, pulsed readout is feasible to improve CNR and recording density in MSR-CAD disks.
\end{abstract}

Index Terms-Carrier to noise ratio (CNR), magnetically induced super-resolution by center aperture detection (MSR-CAD), pulsed-laser readout.

\section{INTRODUCTION}

$\mathbf{M}$ AGNETICALLY induced superresolution (MSR) by center aperture detection (CAD) [1]-[4] can produce a sub- $\mu \mathrm{m}$ sized aperture to read "below-diffraction-limited" recording marks on magneto-optical (MO) disks. When MSRCAD disks are spinning at high velocity, the aperture produced by dc laser irradiation is not circular but tear-drop in shape, thus complicating signal processing for the asymmetric readout signal [5]-[7]. The pulsed-laser readout scheme [6], [7] was therefore proposed to improve the readout characteristics of MSR-CAD disks. By applying thirty-nanosecond pulsed-laser irradiation, the created quasi-circular aperture can detect sub$\mu \mathrm{m}$ sized recording marks with higher carrier-to-noise ratio (CNR) [5].

The recording and readout characteristics of MSR-CAD disks, such as aperture size, wall width, and noise sources, are dependent upon laser power, pulse duration, timing synchronization, and magnetic interaction of multilayers [4]-[6]. In this paper, we shall report experimental and simulated results of pulsed and do schemes to evaluate the readout characteristics of MSR-CAD disks. The feasibility of pulsed readout is then assessed for MSR-CAD MO disks.

Manuscript received August 12, 1998; revised March 4, 1999. This work was supported by the National Science Council of the Republic of China under Contract NSC 87-2622-E009-006.

B.-M. Chen, B.-N. Yang, W.-K. Hwang, and H.-P. D. Shieh are with the Institute of Electro-Optical Engineering, National Chiao Tung University, Hsinchu, Taiwan, 30010 R.O.C. (e-mail: hpshieh@cc.nctu.edu.tw).

T.-E. Shieh is with the Department of Materials Science and Engineering, National Chiao Tung University, Hsinchu, Taiwan, 30010 R.O.C

Publisher Item Identifier S 0018-9464(99)07906-6.

\begin{tabular}{|c|}
\hline SiNx $30 \mathrm{~nm}$ \\
DyFeCo $50 \mathrm{~nm}$ \\
\hline SiNx $2 \mathrm{~nm}$ \\
\hline GdFeCo $50 \mathrm{~nm}$ \\
SiNx $80 \mathrm{~nm}$ \\
PC Substrate $1.2 \mathrm{~mm}$ \\
\hline
\end{tabular}

Fig. 1. MSR-CAD disk structure.

\section{Simulation}

In MSR-CAD disks, the readout characteristics are influenced greatly by the temperature profile resulting from laser irradiation on a spinning disk. We used the heat diffusion equation [4], [7], [8] to simulate the temperature profile after the laser irradiation throughout an MSR-CAD disk, and its disk structure is shown in Fig. 1. The physical parameters [3], [6], [7] of the thin film materials used in this study are listed in Table I. A laser source of $785 \mathrm{~nm}$ wavelength, an objective of NA $=0.55$, and a disk linear velocity of $6.6 \mathrm{~m} / \mathrm{s}$ were assumed in the simulation.

In readout process, laser light creates an effective aperture on the GdFeCo readout layer to detect sub- $\mu \mathrm{m}$ marks recorded on the DyFeCo recording layer [1]-[7]. The readout characteristics of MSR-CAD disks are determined by the temperature dependence of Kerr angle of the readout layer $\theta_{K}(T)$ as shown in Fig. 2(a). The simulated temperature profiles and the effective apertures created by a pulsed laser $(4 \mathrm{~mW}, 30 \mathrm{~ns})$ and a dc laser $(3 \mathrm{~mW})$ were shown in Fig. 2(b) for comparison. The effective aperture size is determined by the temperature $T_{m}$ where its Kerr angle is $0.5 \theta_{K \mid \mathrm{MAX}}$, and $\theta_{K \mid \mathrm{MAX}}$ is defined as the maximum value of $\theta_{K}(T)$. In both cases, the simulated aperture size is about $0.5 \mu \mathrm{m}$. To create apertures of the same size, pulsed-laser readout scheme requires a higher laser power than $\mathrm{dc}$ laser readout scheme.

The aperture wall is a kind of noise sources for readout signal [5]-[9]. The temperatures $T_{r 1}$ and $T_{r 2}$, at outer and inner edges of the aperture wall [see Fig. 2(a)], are defined where their Kerr angles are $0.2 \theta_{K \mid \mathrm{MAX}}$ and $0.9 \theta_{K \mid \mathrm{MAX}}$, respectively. $T_{r 1}$ and $T_{r 2}$ of the MSR-CAD disk in discussion are about $110^{\circ}$ and $140^{\circ} \mathrm{C}$, respectively, and thus the transition temperature of $0.2-0.9 \theta_{K \mid \mathrm{MAX}}, \Delta T=T_{r 2}-T_{r 1}$, 
TABLE I

THIN FILM PARAMETERS

\begin{tabular}{c|c|c|c|c|c}
\hline $\begin{array}{l}\text { Parameter } \\
\text { Materials }\end{array}$ & $\begin{array}{c}\text { Refractive } \\
\text { index } \\
\mathrm{n}-\mathrm{i} \mathrm{k}\end{array}$ & $\begin{array}{c}\text { Specific heat } \\
\mathrm{C} \\
\left(\mathrm{J} / \mathrm{cm}^{3} /^{\circ} \mathrm{C}\right)\end{array}$ & $\begin{array}{c}\text { Heat conductivity } \\
\mathrm{K} \\
\left(\mathrm{J} / \mathrm{cm} /{ }^{\circ} \mathrm{C} / \mathrm{s}\right)\end{array}$ & $\begin{array}{c}\text { Curie temp. } \\
\mathrm{T}_{\mathrm{C}} \\
\left({ }^{\circ} \mathrm{C}\right)\end{array}$ & $\begin{array}{c}\text { Compensation temp. } \\
\mathrm{T}_{\text {comp }} \\
\left.{ }^{\circ} \mathrm{C}\right)\end{array}$ \\
\hline $\mathrm{SiNx}$ & $2-\mathrm{i} 0$ & 2 & 0.018 & & \\
\hline $\mathrm{GdFeCo}$ & $3.0-\mathrm{i} 4.2$ & 2.3 & 0.15 & 360 & 270 \\
\hline DyFeCo & $3.6-\mathrm{i} 4.4$ & 2.5 & 0.15 & 260 & 20 \\
\hline Poly-carbonate & $1.5-\mathrm{i} 0$ & 1.8 & 0.002 & & \\
\hline
\end{tabular}

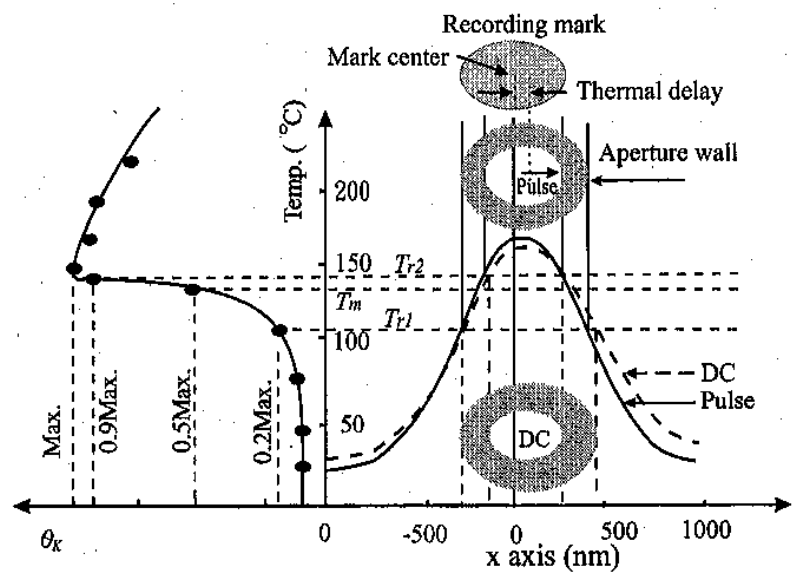

(a)

(b)

Fig. 2, (a) Experimental $\theta_{K}(T)$ and (b) simulated temperature profile and effective aperture of an MSR-CAD disk.

is about $30^{\circ} \mathrm{C}$. The transition noise [9] was reported to be proportional to $20 \times \log (d)$ where the aperture wall width $d$ denoted the distance between the isotherms of $T_{r 1}$ and $T_{r 2}$. As shown in Fig. 2(b), the temperature profile created by pulsed irradiation is more circular, sharper, and nearer to the beam center than that created by dc irradiation. The wall width $d$ is determined to be about 0.18 and $0.25 \mu \mathrm{m}$, for pulsed and dc irradiation, respectively, implying that the aperture wall width in pulsed readout is narrower than that in de readout. Accordingly, the noise level induced in pulsed-laser readout scheme is of about $2.8 \mathrm{~dB}$ lower than in dc readout scheme. Therefore, pulsed-laser readout scheme would be promising to improve the readout signal for MSR-CAD disks when laser power level and pulse duration are optimized.

\section{EXPERIMENTAL}

The fabrication and characterization processes of the MSRCAD disks were the same as reported in the previous article [4]. The schematics of the optical disk tester used to measure the required bias field $(H b)$ and CNR by light intensity modulation is shown in Fig. 3. The synchronous signal from the readout channel of the tester, as shown in Fig. 4, triggered the pulse generator to produce a pulse train for data recording and pulsed readout. The RF signal was measured by a digital oscilloscope whose timing of data acquisition was determined by the writing/erasing control signal. CNR was derived from the fast Fourier transform (FFT) of the RF signal.

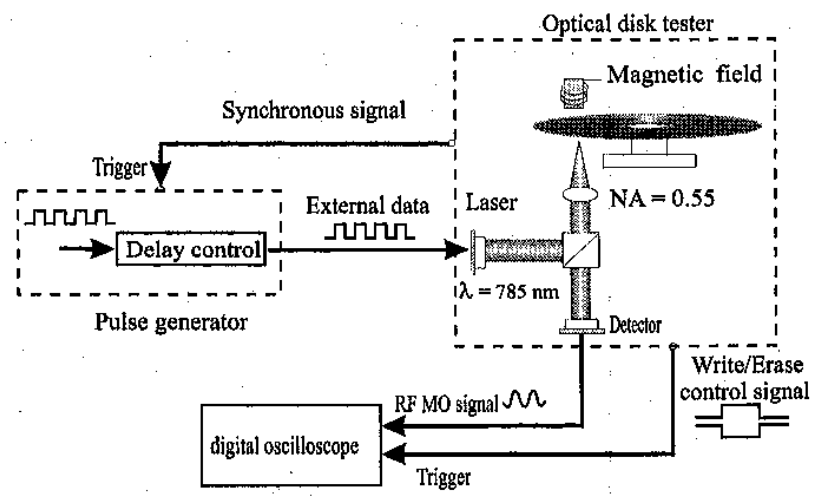

Fig. 3. Schematics of the disk tester.

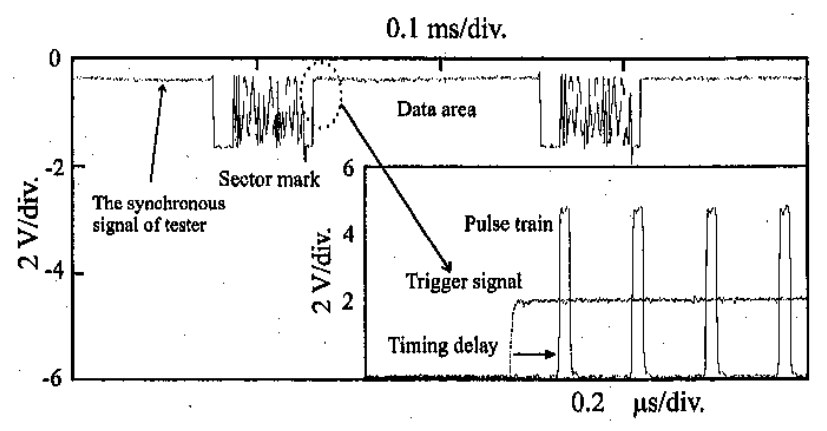

Fig. 4. Trigger signal, pulse train, and timing delay,

The variations of recording and readout parameters, such as laser power, pulse duration, external magnetic field, and complicated multilayered magnetic interactions, may result in timing/spatial offset between recorded marks and effective aperture. Difference in laser power level, pulse duration, and even disk velocity and structure would cause different degrees of thermal delay, as revealed in Fig. 2(b). Here, thermal delay is the timing/spatial deviation between the recorded mark and effective aperture. As shown in Fig. 4, a delay control thus should be added to synchronize the read/write channel clocks for pulsed laser readout.

The polarity of RF signal depends upon the magnetization orientation of the recording layer. For example, the polarity of RF signal is positive due to the upward magnetization of the recording layer, and negative for downward magnetization as shown in Fig. 5(a) and (b), respectively. Actual data stream consists of upward and downward magnetizations. Thus, a double-frequency scheme, where pulsed laser readout frequency is twice the writing frequency, is used to detect the recorded data accurately. 


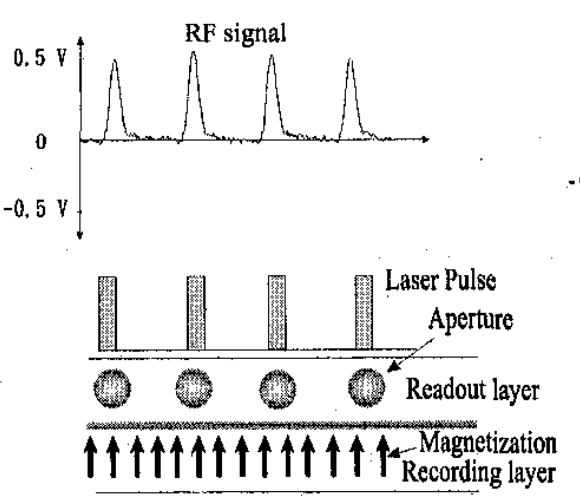

(a)
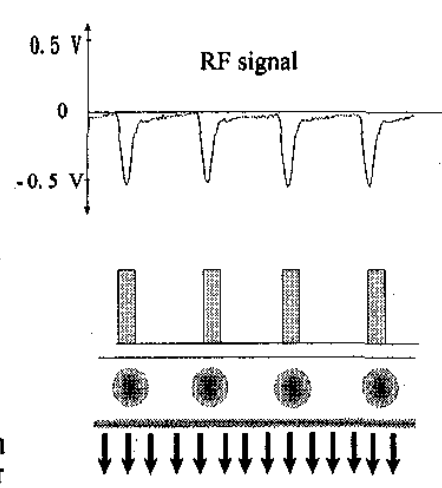

(b)
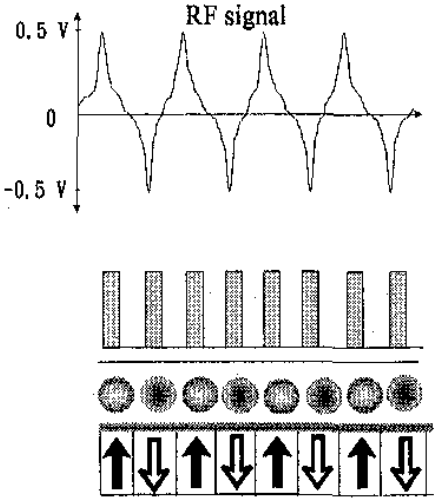

(c)

Fig. 5. Pulsed laser readout signals for: (a) upward, (b) downward magnetization, and (c) recorded data.

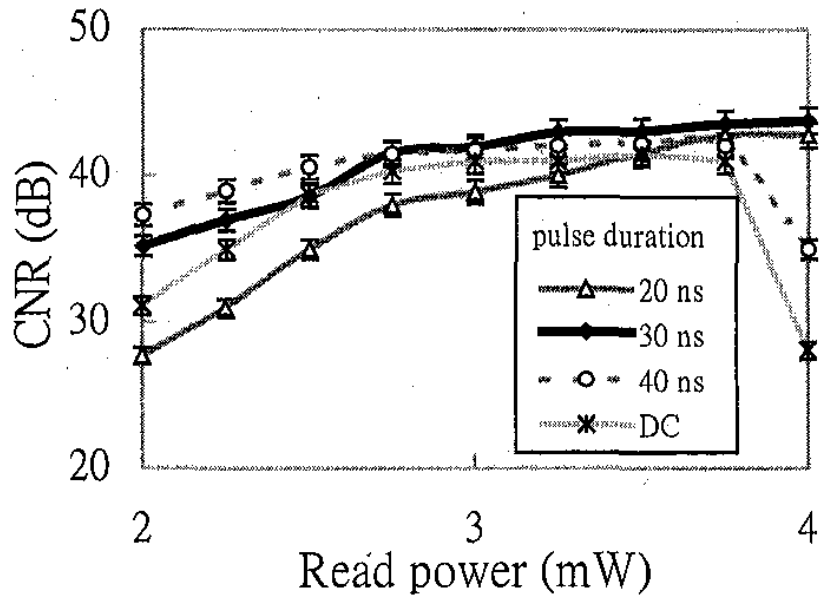

Fig. 6. CNR dependence on pulse duration and read power of the MSR-CAD disk at $0.5 \mu \mathrm{m}$ mark size.

\section{RESULTS AND DISCUSSION}

\section{A. CNR Versus Pulse Duration}

The effective aperture size and position of MSR-CAD disks using pulsed-laser readout scheme are determined by laser power level, pulse duration, and timing offset. The CNR dependence on pulse duration and read power level of the MSR-CAD disk is shown in Fig. 6, where mark size is 0.5 $\mu \mathrm{m}$ and the writing conditions are 6-mW of write power, $60-$ $\mathrm{ns}$ of pulse width, and 100-Oe of bias field. In dc readout, CNR increases with read power $\left(P_{r}\right)$ at $P_{r}<2.75 \mathrm{~mW}$, then increases slightly before $P_{r}$ reaches $3.75 \mathrm{~mW}$. According to the simulation, $3 \mathrm{~mW} \mathrm{dc}$ laser power would create a $0.5 \mu \mathrm{m}$ aperture. Thus, when $P_{r}<3 \mathrm{~mW}$, the aperture is proportional to $P_{r}$, but still less than the recorded mark size of $0.5 \mu \mathrm{m}$. When the laser power is over $3 \mathrm{~mW}$, the created aperture is larger than the recorded mark size, and thus the optical resolvability is reduced. Moreover, as shown in Fig. 2(a), $\Theta k$ decreases with increasing temperature when temperature is over $140^{\circ} \mathrm{C}$. Since readout signal is proportional to readout power, CNR increases slowly at $P_{r}>3 \mathrm{~mW}$. Then, at $P_{r} \geq 3.75 \mathrm{~mW}$, the recorded marks are destroyed and CNR decreases drastically.

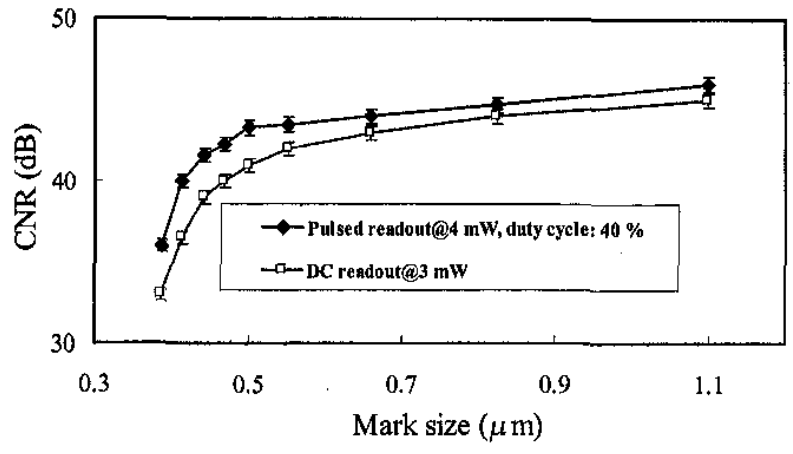

Fig. 7. CNR dependence on recording mark size of an MSR-CAD disk.

Pused readout can improve CNR with suitable pulse width and power level. For pulse duration of 30 or $40 \mathrm{~ns}$, the CNR's are higher than that of de readout when $P_{r} \geq 2.5 \mathrm{~mW}$, as shown in Fig. 6. Pulsed readout of about $2.5 \mathrm{~dB}$ higher than de readout in CNR is achieved when the laser power and pulse duration are $4 \mathrm{~mW}$ and $30 \mathrm{~ns}$, respectively. A similar effect could be observed in pulsed duration of $20 \mathrm{~ns}$ when $P_{r} \geq 3.5$ $\mathrm{mW}$. The reason why CNR depends on laser power is the same as in the above discussions for dc readout. Thus, CNR can be significantly improved by pulsed readout when laser power and pulse duration are optimized for the MSR-CAD MO disks.

\section{B. Mark Size Versus CNR}

CNR and readout signal are generally proportional to the recording mark size. CNR dependence of the recording mark size for the MSR-CAD disk readout by pulsed-laser of $4 \mathrm{~mW}$ and dc readout of $3 \mathrm{~mW}$ is shown in Fig. 7. The results of Fig. 7 showed that CNR's of pulsed-laser readout were always higher than that of dc-readout. Especially, for the mark size ranging from $0.4-0.65 \mu \mathrm{m}$, CNR's of the former are of about $2.5 \mathrm{~dB}$ higher than that of the latter. The experimental results agreed with the simulation.

To reveal the reason why the CNR is improved by pulsed laser readout, Fig. 8 shows the power spectrum of pulsed and dc-laser readouts at mark size of $0.47 \mu \mathrm{m}$. The carrier level of pulsed laser readout is about $1 \mathrm{~dB}$ lower than that of $\mathrm{dc}$ readout, but the noise level of the former is about $3.5 \mathrm{~dB}$ lower than 


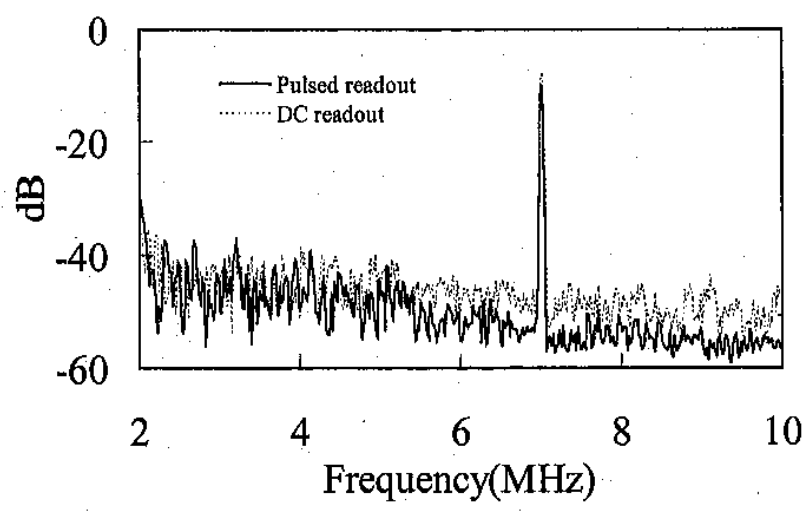

Fig. 8. Power spectrum of pulsed- and dc-laser readouts at a mark size of $0.47 \mu \mathrm{m}$.

that of the latter. Thus, the improved CNR of pulsed laser readout mainly results from the reduction of aperture wall width and/or the suppression of transition noise and recording mark transition noise during readout. The minimum achievable size of well-defined MO mark is limited to about $0.45 \mu \mathrm{m}$ by light intensity modulation with 785-nm laser wavelength in our optical disk tester. Since the required bias field of the MSR-CAD disk is only $100 \mathrm{Oe}$, magnetic field modulation direct overwrite [10] can be applied to produce recorded mark sizes of less than $0.45 \mu \mathrm{m}$. Thus, pulsed laser readout can improve the readout signal and increase recording density in MSR-CAD disks.

Based on the above discussions, pulsed laser readout can produce a symmetric, well-defined aperture, and thus lower thermal crosstalk, aperture wall noise, and recording mark transition noise. Accordingly, the improvements on CNR and reduction of thermal crosstalk for MSR-CAD disks by pulsed laser readout allow the track-pitch and the mark size to be reduced further. Therefore, pulsed laser readout is suitable for MSR-CAD disks for high density recording.

\section{CONCLUSIONS}

Pulsed-laser readout has been demonstrated to improve the readout characteristics of MSR-CAD disks by both simulation and experiments. The aperture generated by pulsed laser irradiation on an MSR-CAD MO disk is more circular and symmetrical than that by de laser irradiation. The narrower aperture wall induced by pulsed laser readout results in lower readout noise "aperture wall noise and recording mark transition noise" than that by dc laser readout. In our experiments, the pulsed laser readout scheme produced CNR about $2.5 \mathrm{~dB}$ higher than that of conventional dc readout with optimized laser power and pulse duration. Therefore, pulsed-laser readout can further improve the readout characteristics, thus potentially leading to higher recording density than dc readout in MSRCAD MO disks.

\section{REFERENCES}

[1] A. Takahashi, J. Nakajima, Y. Murakami; K. Ohta, and T. Ishikawa, "Improvement of readout resolution with an in-plane magnetization film for a magneto-optical disk," IEEE Trans. Magn., vol. 30, pp, 232-236, 1994.

[2] Y. Murakami, A. Takahashi, and S. Terashima, "Magnetic superresolution," IEEE Trans. Magn., vol. 31, pp. 3215-3220, 1995.

[3] K. Torazawa, S. Sumi, S. Yonezawa, N. Suzuki, Y. Tanaka, A. Takahashi, Y. Murakami, and N. Ohta, "A study on key technologies to realize magneto-optical storage of over 7 GBytes in $\mathrm{CD}$ size disk," IEICE Trans. Electron.; vol. E80-C, no. 9, pp. 1142-1148, 1997.

[4] W. K. Hwang, J. R. Liu, and H. P. D. Shieh, "Interface wall energy in exchange-coupled magneto-optical recording films," Jpn. J. Appl. Phys., vol. 36, pp. 2715-2720, 1997

[5] S. Yonezawa and $M$. Takahashi, "Thermodynamic simulation of magnetic-field modulation methods for pulsed laser irradiation in magneto-optical disks," Appl. Opt., vol. 33, pp. 2333-2337, 1994.

[6] B. W. Yang, W. K. Hwang, and H. P. D. Shieh, "Readout scheme by pulsed irradiation center aperture detection on magnetically induced super resolution magnetooptical disks,".Jpn. J. Appl. Phys., vol. 35, pp. $419-422,1996$

[7] S. Yonezawa and H.-P. D. Shieh, "Using laser pulsed for readout in optical recording," Taiwan Patent \#107, 340, Dec, 1994.

[8] M. Mansuripur, G. A. N. Connell, and J. W. Goodman, "Laser-induced local heating of multilayers," Appl. Opt, vol. 21, pp. 1106-1114, 1982.

[9] C.-J. Lin, "High carrier-to-noise ratio achieved on magneto-optic recording disks using a plurality of magneto-optic recording layers," Appl. Phys. Lett., vol. 62, pp. 636-638, 1993.

[10] K. Tsutsumi and T. Fukami, "Direct overwrite in magneto-optic recording," J. Magn. Magn. Mater., vol. 118, pp. 231-247, 1993. 